\title{
On the projection of the presupposition of embedded questions*
}

\author{
Wataru Uegaki \\ Leiden University
}

\begin{abstract}
The projection patterns of the existential/uniqueness presupposition of a $w h$-complement vary depending on the predicate that embeds it. This variation poses problems for existing accounts that treat the presupposition as a semantic contribution of an operator merging with the wh-complement (Dayal 1996) or of the embedding predicate (Uegaki 2015). Rather, the patterns suggest an analysis that treats the presupposition as contributed by the propositions corresponding to the answers of the embedded question.
\end{abstract}

Keywords: embedded questions, complementation, presupposition projection, the presupposition of $w h$-complements.

\section{Introduction}

In recent years, there has been a renewed interest in the semantic analysis of embedded questions (e.g., Spector \& Egré 2015; Uegaki 2015; Cremers 2016; Xiang 2016; Theiler, Roelofsen \& Aloni 2018), following earlier pioneering works (e.g., Karttunen 1977; Groenendijk \& Stokhof 1984; Heim 1994; Dayal 1996; Lahiri 2002). One of the primary goals of the investigation in this domain is to provide a unified analysis of the interpretations of pairs of sentences of the form in (1), where $Q$ is an interrogative and $p$ is a declarative complement, across different predicates $V$ :

$$
\begin{array}{ll}
\text { a. } x \text { Vs } Q & \text { (e.g., Max knows who danced.) } \\
\text { b. } x \text { Vs that } p & \text { (e.g., Max knows that Pat danced.) }
\end{array}
$$

This paper aims to further advance the investigation into the semantics of these constructions, focusing on an issue concerning their presuppositions, i.e., how the presupposition of a $w h$-complement is projected by different embedding predicates.

At least since Katz \& Postal 1964, it has been frequently observed that whquestions in general presuppose that at least one of its Hamblin answers (Hamblin 1973 ) is true. The following sentences exemplify this observation:

* I would like to thank Lucas Champolion, Ivano Ciardelli, Alexandre Cremers, Jakub Dotlačil, Danny Fox, Irene Heim, Tom Roberts, Floris Roelofsen, Benjamin Spector, Yasu Sudo, Mitcho Erlewine and Nadine Theiler for helpful comments and discussion. 
a. Who smokes? $\stackrel{\text { presup }}{\Rightarrow}$ Someone smokes.

$(\stackrel{\text { presup }}{\Rightarrow}:$ 'presupposes')

b. Which semanticists danced? $\stackrel{\text { presup }}{\Rightarrow}$ Some semanticist danced.

In addition, it is well-known that a singular-which question of the form $\ulcorner$ Which NP $\varphi$ ? $\urcorner$ presupposes that exactly one NP $\varphi$ (e.g., Dayal 1996; cf. also Groenendijk \& Stokhof 1984):

(3) Which semanticist danced? $\stackrel{\text { presup }}{\Rightarrow}$ Exactly one semanticist danced.

What is less investigated is what happens to the presupposition when the whquestion is embedded by different predicates, as in the following examples:

(4) a. Max knows which semanticist danced.

b. Max is certain (about) which semanticist danced.

c. Max agrees with Kim on which semanticist danced.

As will be argued below, the projection patterns of the presupposition of the whcomplement in sentences like (4) pose problems for existing accounts. The goal of the paper is thus to provide an alternative analysis of the presupposition of $w h$ complements that correctly captures its projection patterns. Specifically, I will argue that the data require a view where the existential/uniqueness presupposition is contributed by the propositions corresponding to the answers of the question, rather than an operator that merges with the wh-complement (Dayal 1996) or the lexical semantics of the embedding predicate (Uegaki 2015).

It is also important to note that the analysis is intended as a part of the overall semantics of question-embedding, as envisioned in the beginning of the paper. For this reason, our account of the presupposition of $\ulcorner x V s Q\urcorner$ has to be compatible with the presupposition of $\ulcorner x V s$ that $p\urcorner$, given a unified account of the interpretations of $\ulcorner x V s Q\urcorner$ and $\ulcorner x V s$ that $p\urcorner$. That is, the ultimate account of the presuppositions of (4) should also account for the presuppositions of the following kind of sentences:

a. Max knows that Ash danced.

b. Max is certain that Ash danced.

c. Max agrees with Kim that Ash danced.

The structure of the rest of the paper is as follows. In Section 2, I lay out the basic data and why they pose problems for two existing accounts: Dayal (1996) and Uegaki (2015). Section 3 sketches the outline of the proposal, where the existential/uniqueness presupposition of $w h$-complements is contributed by the answer propositions. Finally, Section 4 presents a compositional implementation. 
On the projection of the presupposition of embedded questions

\section{Data and problems for existing accounts}

In this section, I present the data concerning the projection of the presupposition of wh-complements, and discuss why they are problematic for the existing accounts by Dayal (1996) and Uegaki (2015). Here and in the rest of the paper, I will mostly present data involving the uniqueness presupposition (UP) of singular-which questions. However, all analyses to be discussed will be compatible with the existential presupposition of plural-which and simplex wh-questions, as they are based on Dayal's (1996) analysis that uniformly treats both UP and existential presuppositions as the maximality presupposition, as discussed at the end of Section 2.1.

\subsection{Embedding under factive predicates and Dayal's (1996) analysis}

When a singular-which question is embedded under a factive predicate, such as know and surprise, the uniqueness presupposition (UP) projects to the matrix level. This can be seen in the following examples:

a. Max doesn't know which student smokes.

b. Does Max know which student smokes?

c. If Max knows which student smokes, he will tell us about it. $\stackrel{\text { presup }}{\Rightarrow}$ 'Exactly one student smokes.'

(7) a. Max isn't surprised (about) which student smokes.

b. Is Max surprised (about) which student smokes?

c. If Max is surprised (about) which student smokes, he will tell us about it. $\stackrel{\text { presup }}{\Rightarrow}$ 'Exactly one student smokes.'

Dayal's (1996) analysis of the UP employing her answerhood operator straightforwardly captures this matrix projection pattern. In Dayal (1996), wh-complements obligatorily merge with the answerhood operator ANS defined below. ${ }^{1,2}$
a. $\operatorname{ANS}_{w}=\lambda Q_{\langle s t, t\rangle}: \exists p \in Q\left[p=\operatorname{MAX}_{\text {inf }}(Q, w)\right] . \operatorname{MAX}_{\text {inf }}(Q, w)$
b. $\operatorname{MAX}_{\text {inf }}(Q, w)=p$ iff $w \in p \wedge \forall q \in Q[w \in q \rightarrow p \subseteq q]$

ANS roughly acts as a definite determiner over propositions. It carries the presupposition that there is a maximally informative true answer in the set of propositions it combines with, and picks out such a maximally informative true answer. Hereafter, I will refer to the presupposition of ANS as the MAXIMALITY PRESUPPOSITION, and the proposition that the ANS-operator returns from a question as the

1 The formulation using the predicate MAX ${ }_{\text {inf }}$ is from Fox \& Hackl (2007).

2 Following Heim \& Kratzer (1998), I model presuppositions in terms of partial functions with the notation $\lambda x: \pi(x) \cdot \varphi(x)$. Here, $\pi(x)$ is the domain specification corresponding to the presupposition. 
DAYAL-ANSWER of the question. Given that a singular-which question denotes a set of mutually-independent 'atomic' answers, as in (9) (cf. Hamblin 1973), this treatment captures the UP associated with it. ${ }^{3}$

$$
\begin{aligned}
& \text { which student smokes } \rightsquigarrow \\
& \left\{\lambda w^{\prime} \cdot \mathbf{s m o k e}_{w^{\prime}}(a), \lambda w^{\prime} \cdot \mathbf{s m o k e}_{w^{\prime}}(b), \lambda w^{\prime} \cdot \mathbf{s m o k e}_{w^{\prime}}(c)\right\} \quad(=Q)
\end{aligned}
$$

This is so because, for every $w$ and every set $Q$ of mutually-independent propositions, $\operatorname{ANS}_{w}(Q)$ is defined only if exactly one of $Q$ 's members is true in $w$. Sentences like (6-7) have a semantic representation like (10), where $\operatorname{ANS}_{w}(Q)$ (with the matrix evaluation world $w$ ) serves as an argument of the embedding predicate.

$$
\text { Max knows which student smokes } \rightsquigarrow \operatorname{know}_{w}\left(\mathbf{m}, \operatorname{ANS}_{w}(Q)\right)
$$

The meaning in (10) is defined only if $\operatorname{ANS}_{w}(Q)$ is defined, which holds just in case exactly one student smokes in $w$, the matrix evaluation world.

The ANS-operator further enables a uniform treatment of the UP of singularwhich questions and the existential presupposition of plural/simplex wh questions, under the assumption that plural/simplex $w h$-phrases are number-neutral. This is so since the maximality presupposition is satisfied for proposition-sets that are closed under conjunction as long as there is a true answer in the set. ${ }^{4}$

\subsection{Embedding under non-factive predicates}

However, the treatment along the lines of Dayal (1996) encounters a problem once we move to embedding under non-factive predicates. The examples in (11) illustrate that, when be certain embeds a singular which-complement, the UP does not necessarily project to the matrix level, but rather projects into the subject's beliefs.

a. Max isn't certain (about) which student smokes.

b. Is Max certain (about) which student smokes?

c. If Max is certain (about) which student smokes, he will let us know.

$\stackrel{\text { presup }}{\Rightarrow}$ 'Exactly one student smokes.'

$\stackrel{\text { presup }}{\Rightarrow}$ 'Max believes that exactly one student smokes.'

The contrast between know and be certain with respect to the relevant presupposition projection behavior is also clear in the following minimal pair. ${ }^{5}$

3 I assume that a linguistic expression is translated into a formula in an intermediate logical language similar to Ty2 (Gallin 1975), which then receives a model-theoretic interpretation. I notate ' $S \rightsquigarrow \varphi$ ' to indicate that the sentence $S$ is translated into the formula $\varphi$.

4 But, see Elliott, Nicolae \& Sauerland (2018) for recent discussion about the difference between simplex wh and which-questions.

5 M. Erlewine (p.c.) pointed out to me that there seems to be a contrast between the projection patterns of the existential implication and the 'less than two' implication in (11). That is, although 
On the projection of the presupposition of embedded questions

(12) No student smokes. But, Max believes that there is a student smoker. Only, he $\{$ isn't certain / \#doesn't know $\}$ which student smokes.

Another non-factive predicate, agree, exhibits a slightly different presupposition projection behavior, as illustrated in the following examples:

a. Max doesn't agree with Kim on which student smokes.

b. Does Max agree with Kim on which student smokes?

c. If Max agrees with Kim on which student smokes, he'll let her know.

$\stackrel{\text { presup }}{\Rightarrow}$ 'Exactly one student smokes.'

$\stackrel{\text { presup }}{\Rightarrow}$ 'Max and Kim believes that exactly one student smokes.'

These examples show that the UP of the embedded question is projected not only to the belief state of the subject (Max in (13)) but also to the belief state of the individual denoted by the with-phrase (henceforth the with-ARGUMENT; Kim in (13)). ${ }^{6}$ The presupposition, however, is not necessarily projected to the matrix level. The examples in (13) can be felicitous even if there is in fact no student smoker.

Dayal's (1996) analysis in terms of ANS outlined above is not designed to deal with embedding under non-factive predicates. A simple extension of the analysis to the non-factive cases would have ANS interpreted with respect to the matrix evaluation world, meaning that the predicted interpretation would involve the 'actual' answer (relative to the evaluation world) regardless of the embedding predicate. Among other issues, such an analysis would incorrectly predict the UP to project to the matrix level even when the embedding predicate is non-factive. For example, the embedding under be certain would be analyzed as follows:

Max is certain about which student smokes $\rightsquigarrow \operatorname{certain}_{w}\left(\mathbf{m}, \operatorname{ANS}_{w}(Q)\right)$

Just as in the case with factive predicates, the predicted meaning in (14) is defined only if $\operatorname{ANS}_{w}(Q)$ is defined, which in turn holds just in case exactly one student smokes in the matrix evaluation world $w$. Thus, this treatment fails to capture the

(11) sounds felicitous when there is in fact no student smoker (as long as Max believes that there is a student smoker), it sounds infelicitous when there are more than one student smokers. If it turns out that this judgment can be replicated systematically, the discussion in this paper should be viewed as concerning only the existential presupposition of wh-complements. At the same time, we would need an independent analysis of the 'less than two' implication that predicts matrix projection.

6 More precisely, the presupposition concerning Kim's beliefs is stronger than what is stated in (13). That is, the sentences presuppose that there is exactly one student such that Kim believes that they smoke. The same does not hold for the presupposition concerning Max's beliefs. Since the weaker formulation of the presupposition in (13) suffices in the illustration of relevant problems for Dayal's (1996) and Uegaki's (2015) analysis to be given below, I will stick to the weaker formulation in this section for the sake of simplicity. I will argue in Section 3.2 that my ultimate analysis captures the asymmetric presuppositions with respect to Max's beliefs and Kim's beliefs in the examples in (13). 
lack of the matrix projection of the UP as well as its projection to the subject's beliefs observed in (11). Similarly, the projection behavior of agree would be problematic. The analysis would treat question-embedding under agree as follows:

Max agrees with Kim on which student smokes $\rightsquigarrow \mathbf{a g r e e}_{w}\left(\mathbf{m}, \mathbf{k}, \operatorname{ANS}_{w}(Q)\right)$

Just as in (14), the analysis predicts the matrix projection, and no projection to the subject's or to the with-argument's beliefs.

In sum, the projection pattern of the UP varies across factive and non-factive predicates. This variation is not captured by a simple extension of Dayal's (1996) analysis, as such an analysis predicts the presupposition triggered by ANS to project to the matrix level regardless of the embedding predicate.

\subsection{Uegaki's (2015) analysis}

Uegaki (2015) provides a solution to the problem pointed out above regarding the contrast between know and be certain, by letting the question-embedding predicates relate the subject's attitude representation to the Dayal-answer of a question in different ways. This is done by treating ANS as a part of the predicate meaning. Specifically, know and be certain are analyzed as follows:

$$
\begin{aligned}
& \text { a. know } \rightsquigarrow \lambda Q_{\langle s t, t\rangle} \lambda x_{e} \cdot \mathbf{k n o w}_{w}\left(x, \operatorname{ANS}_{w}(Q)\right) \\
& \text { b. be certain } \rightsquigarrow \lambda Q_{\langle s t, t\rangle} \lambda x_{e} . \forall v\left[v \in \operatorname{Dox}_{x}^{w} \rightarrow \operatorname{certain}_{w}\left(x, \operatorname{ANS}_{v}(Q)\right)\right]
\end{aligned}
$$

Let us consider the predictions of these analyses in turn. In (16a), know is analyzed as a question-taking predicate that relates an individual to the Dayal-answer of a question. Thus, this treatment derives the same interpretation for a sentence like Max knows which student smokes as the Dayal-style analysis does in (10). Thus, the analysis preserves Dayal's correct prediction that UP projects to the matrix level.

On the other hand, be certain in (16b) predicts something different from the Dayal-style analysis. According to (16b), $x$ is certain (about) $Q$ is true iff for all worlds compatible with $x$ 's beliefs, $x$ is certain that the Dayal-answer of $Q$ in that world is true. The following exemplifies the treatment of the embedding of singularwhich complement according to this analysis:

$$
\begin{aligned}
& \text { Max is certain which student smokes } \rightsquigarrow \\
& \forall v\left[v \in \mathbf{D o x}_{\mathbf{m}}^{w} \rightarrow \operatorname{certain}_{w}\left(\mathbf{m}, \operatorname{ANS}_{v}(Q)\right)\right]
\end{aligned}
$$

The interpretation given in (17) roughly states that Max believes that he knows which student smokes. This matches the intuitive interpretation of certain-wh. In particular, it captures the obligatory strong exhaustivity of a question embedded under be certain, as Uegaki (2015) points out (see Theiler et al. 2018 for a similar treatment of be certain in terms of the notion of introspection). 
On the projection of the presupposition of embedded questions

What is important for our purposes is the fact that the analysis captures the projection pattern of the UP with be certain. Assuming universal projection out of universal quantification, (17) is defined only if $\forall v\left[v \in \mathbf{D o x}_{\mathbf{m}}^{w} \rightarrow \operatorname{ANS}_{v}(Q)\right.$ is defined]. This holds only if Max believes that exactly one student smokes. What is crucial here is the fact that the world with respect to which ANS is evaluated is not the matrix evaluation world, but is bound by the universal quantification over the subject's belief worlds. This is made possible in Uegaki (2015) by letting ANs be part of the lexical semantics of the embedding predicate, rather than an independent operator that feeds a propositional argument to the embedding predicate, as in Dayal (1996).

The presupposition projection behavior of agree discussed in the previous section remains to be a problem for Uegaki (2015) since it is not straightforward to define a plausible lexical entry for agree along the lines of (16) that would derive the projection behaviors. Furthermore, as we will see in the next subsection, even if such a lexical entry were possible, the analysis would face problems when it is extended to declarative complements.

\subsection{Problems for Uegaki (2015)}

Despite the advantages, Uegaki's (2015) analysis faces problems when viewed as a part of the general semantic theory of question-embedding, which would encompass a unified account of the presuppositions of $\ulcorner x V s Q\urcorner$ and $\ulcorner x$ Vs that $p\urcorner$ across different predicates $V$, as envisioned in Section 1 . This is so since extending the analysis to the declarative-embedding cases would make empirically incorrect predictions. To illustrate the problems, we first have to make it explicit how Uegaki's (2015) treatment of interrogative complements embedded under know and be certain can be integrated with an analysis of declarative complements.

Uniform semantics of complementation Uegaki's (2015) analysis is based on the UNIFORM SEMANTICS OF COMPLEMENTATION, where declarative and interrogative complements share the same semantic type, i.e., a set of propositions, which is selected by clause-embedding predicates such as know and be certain (see also Theiler et al. 2018). ${ }^{7}$ In particular, Uegaki (2015) analyzes declarative complements as denoting the singleton set consisting of the proposition it traditionally denotes. For instance, the declarative complement that Ash smokes is analyzed as follows, where $A$ is the proposition that Ash smokes.

$$
\text { that Ash smokes } \rightsquigarrow\{A\}
$$

7 Selectional restrictions of predicates like believe and wonder are analyzed as arising from the semantic incompatibility between their lexical semantics and the particular complement type. See e.g., Ciardelli \& Roelofsen (2015); Uegaki (2015); Theiler, Roelofsen \& Aloni (2017). 
Given this, declarative-embedding under know is analyzed as follows:

$$
\text { Max knows that Ash smokes } \rightsquigarrow \mathbf{k n o w}_{w}\left(\mathbf{m}, \operatorname{ANS}_{w}(\{A\})\right)
$$

The interpretation in (19) presupposes that Ash smokes, and asserts that Max knows that Ash smokes. Here, the presupposition of ANS boils down to the factivity presupposition that Ash smokes. ${ }^{8}$ This is an empirically correct prediction.

Problem 1: be certain that A problem arises when we consider declarativeembedding under be certain. The following is the interpretation predicted by Uegaki (2015) for a sentence with be certain and a declarative complement.

$$
\text { Max is certain that Ash smokes } \rightsquigarrow \forall v\left[v \in \mathbf{D o x}_{\mathbf{m}}^{w} \rightarrow \operatorname{certain}_{w}\left(\mathbf{m}, \operatorname{ANS}_{v}(\{A\})\right)\right]
$$

Assuming a universal projection of presuppositions out of universal quantification (just as in the case of (17) above), we have that (20) presupposes $\forall v\left[v \in \mathbf{D o x}_{\mathbf{m}}^{w} \rightarrow\right.$ $\operatorname{ANS}_{v}(\{A\})$ is defined]. Since $\operatorname{ANS}_{v}(\{A\})$ is defined only if $A(v)$, this presupposition amounts to $\forall v\left[v \in \mathbf{D o x}_{\mathbf{m}}^{w} \rightarrow A(v)\right]$, i.e., Max believes that Ash smokes. Empirically, this presupposition seems too strong for (20). Rather than presupposing Max's belief that Ash smokes, (20) seems to presupposes that it is merely compatible with Max's beliefs that Ash smokes.

Problem 2: agree that The second problem concerns agree. As discussed in Section 2.2, the presupposition of a which-question under agree projects both to the subject's and to the with-argument's beliefs, as illustrated below:

Does Max agree with Kim on which student smokes?

i. $\stackrel{\text { presup }}{\Rightarrow}$ 'Max believes that exactly one student smokes.'

ii. $\stackrel{\text { presup }}{\Rightarrow}$ 'Kim believes that exactly one student smokes.'

An analysis along the lines of Uegaki (2015) would capture this projection behavior by defining a lexical entry for agree in terms of ANS that derives the following:

$$
\ulcorner x \text { agrees with y on } Q\urcorner \text { presupposes: }
$$

i. $x$ believes that $\operatorname{ANS}_{w}(Q)$ is defined. $\Leftrightarrow x$ believes that $Q$ has a strongest true member.

8 Note that it is reasonable to assume that the predicate know in the intermediate language also presupposes the truth of the propositional argument. In such a case, the factivity is redundantly stated in (19), once by the factivity of know and once by ANS. See Uegaki (2015) for a formulation where the attitude predicate in the intermediate language corresponding to know is non-factive, and thus the factivity is solely contributed by ANS. 
On the projection of the presupposition of embedded questions

ii. $y$ believes that $\operatorname{ANS}_{w}(Q)$ is defined.

$\Leftrightarrow y$ believes that $Q$ has a strongest true member.

As briefly mentioned in the end of the previous section, it is not straightforward to define a plausible lexical entry for agree that derives these presuppositions. What is more crucial is that, regardless of whether such a lexical entry can be defined, (22) would make incorrect predictions about the presuppositions of the declarativeembedding under agree. This is so since we would have the following as the declarative-embedding case where $Q$ is a singleton set:

$\ulcorner x$ agrees with y that $p\urcorner$ presupposes:

i. $x$ believes that $\{p\}$ has a strongest true member. $\Leftrightarrow x$ believes $p$.

ii. $y$ believes that $\{p\}$ has a strongest true member. $\Leftrightarrow y$ believes $p$.

This is an incorrect prediction, as an agree-that sentence does not presuppose that the subject believes the complement, as can be seen in the following example:

Does Max agree with Kim that Ash smokes?

$\stackrel{\text { presup }}{\Rightarrow}$ Kim believes that Ash smokes.

$\stackrel{\text { presup }}{\Rightarrow}$ Max believes that Ash smokes.

Taking a step back, both with be certain and with agree, we have seen that the UP projecting to the subject's belief poses a problem for Uegaki (2015). The core of the problem is the same across the two predicates. Even if the analysis correctly predicts the projection behavior in the interrogative case, it incorrectly predicts that a similar pattern would hold for the declarative case. This is by virtue of the fact that the presupposition is encoded in the lexical semantics of the embedding predicate and that declarative complements are treated as singleton proposition-sets. ${ }^{9}$

9 It is in principle possible to define a lexical entry for the relevant predicates that avoids this issue, by making the entry sensitive to the cardinality of the proposition-set in the first argument. For example, the following lexical entry for be certain captures the presupposition projection behavior in the interrogative-embedding case while avoiding the problematic prediction in the declarativeembedding case:

$$
\text { be certain } \rightsquigarrow \lambda Q_{\langle s t, t\rangle} \lambda x_{e} \cdot\left[\begin{array}{l}
|Q|>1 \rightarrow \forall v\left[v \in \operatorname{Dox}_{x}^{w} \rightarrow \operatorname{certain}_{w}\left(x, \operatorname{ANS}_{v}(Q)\right)\right] \\
|Q|=1 \rightarrow \operatorname{certain}_{w}(x, \cup Q)
\end{array}\right]
$$

However, it is plausible to assume that there is a general constraint against a lexical entry that is sensitive to the cardinality of the proposition-set. This is so, since allowing such lexical entries leads to the danger of ruling in various empirically implausible lexical entries. One such lexical entry is *shknow discussed by Spector \& Egré (2015), which would mean 'know' with declarative complements and 'wonder' with interrogative complements. See George (2011: §4.5.2), Theiler et al. (2018: §5) and Uegaki (to appear: §6) for related discussion. 


\subsection{Diagnosing the problems}

So far, I have considered two existing analyses concerning the uniqueness/existential presupposition of wh-complements, i.e., Dayal (1996) and Uegaki (2015), and argued that neither can fully capture the projection patterns with different embedding predicates. In this section, I will state the problem in more general terms.

Abstractly, we can understand the difference between Dayal (1996) and Uegaki (2015) as the difference in the locus of the presupposition-carrier, i.e., which lexical item is defined as a partial function. In Dayal 1996, the relevant presupposition is triggered by the ANS-operator. On the other hand, in Uegaki 2015, it is triggered by the embedding predicate (since the ANS-operator is part of the predicate's lexical semantics). This is schematically represented in (25), where the boxes mark the items that trigger the presupposition.

$$
\begin{aligned}
& \ulcorner x \text { Vs } Q\urcorner \rightsquigarrow \\
& \text { (i) } V(x, \text { ANS }(Q)) \\
& \text { (ii) } V(x, Q)
\end{aligned}
$$

In (i), ANS is defined as a partial function that triggers the maximality presupposition. The application of ANS to $Q$ is defined if this presupposition is met with respect to the matrix evaluation world and $Q$, and the proposition resulting from the application does not carry the presupposition. The problem with this treatment is that it incorrectly predicts the presupposition to project to the matrix level regardless of the embedding predicate $V$.

In (ii), the maximality presupposition is encoded in the lexical semantics of the predicate in ways that vary across predicates, deriving lexically-specific projection patterns. As such, this treatment overcomes the problem with the Dayal-style analysis in (i). However, it encounters another problem, i.e., it makes an incorrect prediction with respect to declarative complements. Since the presupposition is encoded in the predicate, the analysis incorrectly predicts that the presupposition shows up with declarative complements as well, assuming that the same lexical entry is used for both interrogative and declarative complementation. The prediction with respect to the declarative complements is not a problem for the Dayal-style analysis schematized in (i) since ANS appears only in interrogative complements.

At this point, the third analytic possibility presents itself: The presupposition can in principle be carried by the complement meaning. We can schematize this possibility as follows:

$$
\ulcorner x V s Q\urcorner \rightsquigarrow V(x, f(Q))
$$

Here, $f$ is a (possibly vacuous) operator such as ANS that takes the question denotation as an input and passes it to the predicate meaning. What is crucial in this 
On the projection of the presupposition of embedded questions

schema is that the semantic argument of the predicate corresponding to the interrogative complement carries the presupposition. Note that this possibility is different from that in (25-i), since $f(Q)$ as a whole in (26) carries the relevant presupposition while $\operatorname{Ans}(Q)$ in (25-i) doesn't.

It is clear at this point that the analysis schematized in (26) does not run into the same problem as the Uegaki-style analysis in (25-ii), i.e., the incorrect prediction with declarative complements, since the presupposition would not be triggered in the case of declarative complementation. The remaining question is whether the analysis can overcome the problem for the Dayal-style analysis in (25-ii), i.e., the variation in the projection patterns across predicates. In the next section, I will argue that the line of analysis in (26) provides a straightforward account of the variation in the projection patterns, once we analyze the answer(s) as carrying the uniqueness/existential presupposition.

\section{The Partial Proposition Analysis}

As suggested in the previous section, I propose that it is the complement meaning that carries the uniqueness/existential presupposition of wh-complements, rather than an operator that outputs an answer proposition (which itself is devoid of the uniqueness presupposition) (Dayal 1996) or a question-embedding predicate (Uegaki 2015). In fleshing out this analysis, it is particularly informative to consider the behavior of other kinds of presuppositions triggered within a wh-complement.

\subsection{Presupposition triggers within an interrogative complement}

Consider example (27), a wh-clause involving a singular definite DP inside it:

who caught the unicorn

Here, it is reasonable to think that each answer in the Hamblin-denotation of this clause carries a presupposition about the unique existence of a unicorn. That is, the Hamblin-denotation of (27) would look like the following:

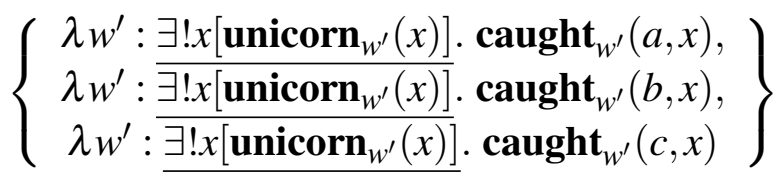

What is crucial for us is that the projection patterns of this presupposition under various embedding predicates behaves exactly like the projection patterns of the UP of singular-which questions observed in the previous section:

a. Max knows who caught the unicorn. $\stackrel{\text { presup }}{\Rightarrow}$ There is a unique unicorn. 
b. Max is certain who caught the unicorn.

$\stackrel{\text { presup }}{\Rightarrow}$ Max believes that there is a unique unicorn.

c. Max agrees with Kim on who caught the unicorn.

$\stackrel{\text { presup }}{\Rightarrow}$ Max and Kim believe that there is a unique unicorn.

As can be seen above, the presupposition that there is a unique unicorn projects to the matrix level with know, to the subject's beliefs with be certain, and both to the subject's and to the with-argument's beliefs with agree.

The parallel in projection patterns in (29) and the UP of singular-which questions is straightforwardly explained if the latter is encoded in each proposition in the Hamblin denotation, just as in (28). That is, which student smokes has the Hamblin-denotation that looks like (30), where each proposition in the set carries the proposition that exactly one student smokes (see Rullmann \& Beck 1998 and Champollion, Ciardelli \& Roelofsen 2017 for existing proposals along these lines regarding the existential presupposition of $w h$-complements).

$$
\begin{aligned}
& \text { which student smokes } \rightsquigarrow \\
& \left\{\begin{array}{c}
\lambda w^{\prime}: \exists ! x\left[\text { student }_{w^{\prime}}(x) \wedge \text { smoke }_{w^{\prime}}(x)\right] . \text { smoke }_{w^{\prime}}(a), \\
\lambda w^{\prime}: \exists ! x\left[\text { student }_{w^{\prime}}(x) \wedge \text { smoke }_{w^{\prime}}(x)\right] . \\
\text { smoke }_{w^{\prime}}(b), \\
\lambda w^{\prime}: \exists ! x\left[\text { student }_{w^{\prime}}(x) \wedge \mathbf{s m o k e}_{w^{\prime}}(x)\right] . \text { smoke }_{w^{\prime}}(c)
\end{array}\right\}
\end{aligned}
$$

Since this analysis treats each answer of a singular-which question as a partial function that presupposes uniqueness, I will hereafter refer to it as the PARTIAL PROPOSITION ANALYSIS. In the next section, I will demonstrate how this analysis captures the projection patterns of the UP observed in Section 2.

\subsection{Deriving the projection patterns}

Once the UP is encoded in each proposition in the Hamblin denotation of a singularwhich question, its projection pattern can be straightforwardly accounted for. In this section, I will demonstrate this with the now-familiar three predicates: know, be certain and agree. In doing so, I will assume two things as given: (i) patterns of presupposition projection from the declarative complements of the three predicates and (ii) the existential semantics for question-embedding (cf. e.g., Spector \& Egré 2015), which can be schematized as follows:

$$
\ulcorner x V s Q\urcorner \exists p \in Q[V(p)(x)]
$$

These two assumptions are made in this section for expository purposes, but they are shown to derive from a concrete lexical semantics of embedding predicates later given in Section 4. Also, although the simple-minded analysis in (31) cannot deal with weakly and strongly exhaustive readings of embedded questions, Section 4 addresses how the exhaustive readings are derived in the compositional analysis. 
On the projection of the presupposition of embedded questions

The presupposition projection in $\boldsymbol{V}$-that The projection patterns of presuppositions triggered within a declarative complement of know, be certain and agree can be independently tested by considering the following kind of examples:

a. Max knows that the unicorn danced.

$\stackrel{\text { presup }}{\Rightarrow}$ There is a unique unicorn $\&$ that it danced $\&$ Max believes that there is a unique unicorn.

b. Max is certain that the unicorn danced.

$\stackrel{\text { presup }}{\Rightarrow}$ Max believes there is a unique unicorn \& it is compatible with Max's beliefs that it danced.

c. Max agrees with Kim that the unicorn danced.

$\stackrel{\text { presup }}{\Rightarrow}$ Both Max and Kim believe that there is a unique unicorn \& Kim believes that it danced.

Schematically, we can write the presupposition projection patterns observed in (32) as follows, where $p_{\pi}$ denotes a proposition $p$ that carries the presupposition $\pi$.
a. $\mathbf{k n o w}_{w}\left(x, p_{\pi}\right)$ is defined iff $\pi(w) \wedge p(w) \wedge \mathbf{D o x}_{w}^{x} \subseteq \pi$
b. $\operatorname{certain}_{w}\left(x, p_{\pi}\right)$ is defined iff $\operatorname{Dox}_{w}^{x} \subseteq \pi \wedge \operatorname{Dox}_{w}^{x} \cap p \neq \varnothing$
c. agree $_{w}\left(x, y, p_{\pi}\right)$ is defined iff $\operatorname{Dox}_{w}^{x} \subseteq \pi \wedge \operatorname{Dox}_{w}^{y} \subseteq \pi \wedge \operatorname{Dox}_{w}^{y} \subseteq p$

We now have all the ingredients in place to account for the projection patterns of the UP of singular-which: (i) the Partial Proposition Analysis, as exemplified in (30), (ii) the projection patterns of presuppositions from $V$-that, i.e., (33), and (iii) the existential semantics for question-embedding, i.e., (31). Below, I will go over the accounts for know, be certain and agree in turn. I will abbreviate the relevant UP as $\pi$, as defined in (34). Together with the convention for notating presuppositions in subscripts, we can now write our Hamblin denotation of a which-question as (35).

$$
\pi:=\lambda w \cdot \exists ! x\left[\operatorname{student}_{w}(x) \wedge \text { smoke }_{w}(x)\right]
$$

which student smokes $\rightsquigarrow\left\{A_{\pi}, B_{\pi}, C_{\pi}\right\}$

$$
(=Q)
$$

Know The matrix projection of the UP with know can be derived as follows, which I will elaborate immediately below:

$$
\begin{aligned}
& \text { Max knows which student smokes. } \rightsquigarrow \\
& \exists p_{\pi} \in Q\left[\mathbf{k n o w}_{w}\left(\mathbf{m}, p_{\pi}\right)\right] \\
& \stackrel{\text {presup}}{\Rightarrow} \exists p_{\pi} \in Q\left[\mathbf{k n o w}_{w}\left(\mathbf{m}, p_{\pi}\right) \text { is defined }\right] \\
& \Leftrightarrow \exists p_{\pi} \in Q\left[\pi(w) \wedge p(w) \wedge \mathbf{D o x}_{\mathbf{m}}^{w} \subseteq \pi\right] \\
& \Leftrightarrow \pi(w) \wedge \exists p_{\pi} \in Q[p(w)] \wedge \mathbf{D o x}_{\mathbf{m}}^{w} \subseteq \pi
\end{aligned}
$$


First of all, the sentence is analyzed as involving the existential quantification over Hamblin answers, according to the existential semantics in (31). Assuming existential presupposition projection out of existential quantification, ${ }^{10}$ the meaning described by this formula is defined only if the third line of (36) is true. Given the presupposition projection pattern of know-that in (33a), the scope of the existential quantification in the third line holds iff $\pi(w) \wedge p(w) \wedge \mathbf{D o x}_{\mathbf{m}}^{w} \subseteq \pi$, i.e., iff the presupposition and the proposition $p$ holds in the evaluation world, and Max believes the presupposition. Among these three conjuncts in the scope of the existential quantification over answers, the first and the third conjuncts are not bound by the existential quantification. Thus, the formula in the penultimate line is equivalent to the formula in the last line. As a result, we derive the presupposition that can be paraphrased as follows (I omit the second conjunct in the last line of (36) since it is entailed by the first conjunct):

'Exactly one student smokes \& Max believes that exactly one student smokes.'

This accounts for the matrix projection of the UP with know. Interestingly, it additionally predicts that the presupposition projects into the subject's beliefs. I will briefly discuss the status of this prediction in Section 5 .

Be certain Similarly, we derive the projection with be certain as follows:

$$
\begin{aligned}
& \text { Max is certain which student smokes. } \rightsquigarrow \\
& \exists p_{\pi} \in Q\left[\operatorname{certain}_{w}\left(\mathbf{m}, p_{\pi}\right)\right] \\
& \stackrel{\text { presup }}{\Rightarrow} \exists p_{\pi} \in Q\left[\operatorname{certain}_{w}\left(\mathbf{m}, p_{\pi}\right) \text { is defined }\right] \\
& \Leftrightarrow \exists p_{\pi} \in Q\left[\mathbf{D o x}_{w}^{\mathbf{m}} \subseteq \pi \wedge \mathbf{D o x}_{w}^{\mathbf{m}} \cap p \neq \varnothing\right] \\
& \Leftrightarrow \operatorname{Dox}_{w}^{\mathbf{m}} \subseteq \pi \wedge \exists p_{\pi}\left[\mathbf{D o x}_{w}^{\mathbf{m}} \cap p \neq \varnothing\right]
\end{aligned}
$$

Each step in this derivation follows the same reasoning as the ones in (36). In the last line, the first conjunct (i.e., 'Max believes that exactly one student smokes') entails the second conjunct (i.e., 'there is a student such that their smoking is compatible with Max's beliefs'). As a result, we derive the following presupposition:

$$
\text { 'Max believes that exactly one student smokes.' }
$$

This is exactly what we observed earlier, i.e., the projection to the subject's beliefs.

10 That is, I assume the following as the definedness condition of the existential quantification in the intermediate logical language:

(i) $\exists x[\varphi(x)]$ is defined iff $\exists x[\varphi(x)$ is defined $]$

Beaver (2001) and Chemla (2009) suggest that this is the empirically correct definedness condition for the translation of the quantifier some. 
On the projection of the presupposition of embedded questions

Agree Finally, here is the derivation in the case of agree:

$$
\begin{aligned}
& \text { Max agrees with Kim on which student smokes. } \rightsquigarrow \\
& \exists p_{\pi} \in Q\left[\mathbf{a g r e e}_{w}\left(\mathbf{m}, \mathbf{k}, p_{\pi}\right)\right] \\
& \stackrel{\text { presup }^{\prime}}{\Rightarrow} \exists p_{\pi} \in Q\left[\mathbf{a g r e e}_{w}\left(\mathbf{m}, \mathbf{k}, p_{\pi}\right) \text { is defined }\right] \\
& \Leftrightarrow \exists p_{\pi} \in Q\left[\mathbf{D o x}_{w}^{\mathbf{m}} \subseteq \pi \wedge \mathbf{D o x}_{w}^{\mathbf{k}} \subseteq \pi \wedge \mathbf{D o x}_{w}^{\mathbf{k}} \subseteq p\right] \\
& \Leftrightarrow \operatorname{Dox}_{w}^{\mathbf{m}} \subseteq \pi \wedge \mathbf{D o x}_{w}^{\mathbf{k}} \subseteq \pi \wedge \exists p_{\pi} \in Q\left[\mathbf{D o x}_{w}^{\mathbf{k}} \subseteq p\right]
\end{aligned}
$$

Again, the steps in the derivation follow the same reasonings as in the previous two cases. The resulting presupposition can be described as follows: ${ }^{11}$

'Max believes that exactly one student smokes \& there is exactly one student such that Kim believes she smokes.'

Not only does this capture the projection of the UP both to the subject's and to the with-argument's beliefs, but it also accounts for the asymmetry in strength between the subject's presupposed belief and the with-argument's presupposed belief, discussed earlier in footnote 6.

\subsection{The matrix case}

In the previous subsection, I have demonstrated that the Partial Proposition Analysis correctly captures the projection patterns of the UP in embedded singular-which questions. Still, a question remains as to how the UP of a matrix singular whichquestions, as exemplified below, arises:

$$
\text { Which student smokes? } \stackrel{\text { presup }}{\Rightarrow} \text { 'Exactly one student smokes.' }
$$

Note that the presupposition does not automatically follow from the Partial Answer Analysis since, according to the analysis, it is the answers rather than the question itself that presuppose uniqueness.

Following Rullmann \& Beck (1998: 226), I assume that matrix questions in general presuppose that at least one of their Hamblin answers is true. Given the UP encoded in the answers, the existential presupposition of a matrix singularwhich question is met only if the UP is met. Hence, the UP of a matrix singularwhich question is captured as the combination of the two presuppositions, one from answers and the other from the question as a whole. ${ }^{12}$

11 The second conjunct (i.e., 'Kim believes that exactly one student smokes') and the third conjunct (i.e., 'there is a student such that Kim believes that she smokes') of the last line of (40) are expressed as a single conjunct ('there is exactly one student such that Kim believes she smokes') in (41).

12 It is not crucial for our purposes whether the existential presupposition of matrix questions is treated as a semantic presupposition, as in Rullmann \& Beck (1998), or a pragmatic presupposition as 


\section{Compositional implementations}

In Section 3, I illustrated how the Partial Proposition Analysis captures the projection patterns of the UP under different embedding predicates, given two assumptions: (i) the presupposition projection behaviors of the predicates with declarative complements and (ii) the existential semantics for question-embedding. In this section, I will propose a compositional implementation of the proposal. This includes lexical semantics of question-embedding predicates that derive the two assumptions above and an internal composition of an interrogative complement that derives a set of partial propositions as the denotation of the wh-complement.

\subsection{The lexical semantics of the question-embedding predicates}

My analysis of question-embedding predicates will follow Uegaki (2015), and assume the uniform semantics of complementation (introduced in Section 2.4). That is, the predicates encode a version of the ANS-operator, and the declarative complement is treated as denoting a singleton proposition-set. Only, the ANS-like operator encoded in the predicate lacks the maximality presupposition. In line with the Partial Proposition Analysis, the maximality presupposition comes from the answers.

Concretely, following Lahiri (2002) and Cremers (2016), I define ANs' as an operator that takes an extra argument $C$ which restricts the set of answers:

$$
\operatorname{ANS}^{\prime}\left(C_{\langle s t, t\rangle}\right):=\lambda Q_{\langle s t, t\rangle}: \exists p \in Q \cap C .\left\{p \in C \cap Q \mid \neg \exists p^{\prime} \in Q \cap C\left[p^{\prime} \subset p\right]\right\}
$$

Given a question $Q$ and a restriction $C$, this operator presupposes that there is an answer in $Q \cap C$, and returns the set of maximally strong answers in $Q \cap C$.

Question-embedding predicates encode $\mathrm{ANS}^{\prime}$ in their meanings with lexically specific restrictors, as in (44). Given $Q$ and $x$, they return true iff $x$ believes an answer in the answer-set provided by $\operatorname{ANS}^{\prime}(C)(Q)$.

$$
\begin{aligned}
& \text { a. know } \rightsquigarrow \lambda Q_{\langle\text {st, } t\rangle} \lambda x_{e} . \exists p\left[p \in \operatorname{ANS}^{\prime}(\{p \mid p(w)\})(Q) \wedge \operatorname{Dox}_{x}^{w} \subseteq p\right] \\
& \text { b. be certain } \rightsquigarrow \\
& \quad \lambda Q_{\langle\text {st }, t\rangle} \lambda x_{e} . \exists p\left[p \in \operatorname{ANS}^{\prime}\left(\left\{p \mid p \cap \mathbf{D o x}_{x}^{w} \neq \varnothing\right\}\right)(Q) \wedge \mathbf{D o x}_{x}^{w} \subseteq p\right] \\
& \text { c. agree with } \rightsquigarrow \\
& \quad \lambda y_{e} \lambda Q_{\langle s t, t\rangle} \lambda x_{e} . \exists p\left[p \in \operatorname{ANS}^{\prime}\left(\left\{p \mid \mathbf{D o x}_{y}^{w} \subseteq p\right\}\right)(Q) \wedge \mathbf{D o x}_{x}^{w} \subseteq p\right]
\end{aligned}
$$

This analysis derives the two assumptions made in the previous section, i.e., the presupposition projection behavior of $V$-that and the existential semantics for questionembedding. In particular, the presupposition projection behavior is summarized in

suggested by Groenendijk \& Stokhof (1984: 30-37). However, the current formulation of a Hamblin denotation qua a set of propositions does not readily lend itself to a semantic treatment of the existential presupposition in terms of partial functions. The pragmatic view, on the other hand, is compatible with the current analysis. 
On the projection of the presupposition of embedded questions

\begin{tabular}{lll}
\hline Predicate & presup. of $\ulcorner x$ Vs that $p\urcorner$ & presup. of $\left\ulcorner x\right.$ Vs that $\left.p_{\pi}\right\urcorner$ \\
\hline know & $p(w)$ & $\pi(w) \wedge p(w) \wedge \operatorname{Dox}_{x}^{w} \subseteq \pi$ \\
be certain & $p \cap \mathbf{D o x}_{x}^{w} \neq \varnothing$ & $p \cap \operatorname{Dox}_{x}^{w} \neq \varnothing \wedge \operatorname{Dox}_{x}^{w} \subseteq \pi$ \\
agree with $y$ & $\operatorname{Dox}_{y}^{w} \subseteq p$ & $\operatorname{Dox}_{y}^{w} \subseteq p \wedge \operatorname{Dox}_{y}^{w} \subseteq \pi \wedge \operatorname{Dox}_{x}^{w} \subseteq \pi$ \\
\hline
\end{tabular}

Table 1 Predicted presuppositions for $\ulcorner x V s$ that $p\urcorner$ and $\left\ulcorner x V s\right.$ that $\left.p_{\pi}\right\urcorner$

Table 1. Since a declarative complement denotes a singleton proposition-set, the existential presupposition of ANS $^{\prime}$ manifests itself in $\ulcorner x V s$ that $p\urcorner$ as shown in the second column of the table. Given that $\operatorname{Dox}_{x}^{w} \subseteq p_{\pi}$ presupposes $\operatorname{Dox}_{x}^{w} \subseteq \pi$, we can further derive the presuppositions of $\left\ulcorner x V s\right.$ that $\left.p_{\pi}\right\urcorner$ as in the third column, which is equivalent to what is assumed in (33) above. As these presuppositions existentially project in the case of $\ulcorner x V s Q\urcorner$, we derive the same projection patterns of the uniqueness/existential presupposition as demonstrated in the previous section.

The analysis also makes reasonable predictions regarding exhaustivity. When $C \cap Q$ is closed under conjunction, the analysis predicts weak exhaustivity, just as an analysis that employs Dayal's (1996) ANs. ${ }^{13}$ If $Q$ is not closed under conjunction, the analysis derives a mention-some reading, in line with the analysis of mentionsome by Fox (2013) and Xiang (2016). Finally, I assume that the strong exhaustivity is derived by an optional point-wise exhaustification of the Hamblin denotation (Nicolae 2013; Fox 2018), which projects the presupposition of the answers.

\subsection{The internal composition of the complement}

We now move on to the internal composition of $w h$-complements, i.e., how to compositionally derive the denotation of the complements where the answers carry the existence/uniqueness presupposition. Broadly speaking, there are two approaches to derive the denotation of a wh-complement in the Partial Proposition Analysis. One is to posit an additional operator that adds the maximality presupposition to the answers in the Hamblin denotation. The other is to encode the presupposition to which-NPs and derive a set of partial propositions through presupposition projection. The latter approach has been adopted by Rullmann \& Beck (1998) and Champollion et al. (2017) in their treatment of the existential presupposition of which-questions. On the other hand, to my knowledge, the former approach has not been explicitly advocated in the previous literature. I will submit an analysis in

13 In the case of be certain, given the lexical semantics of the predicate, the weakly exhaustive reading would be equivalent to the strongly exhaustive reading. This is an aspect of the analysis preserved from Uegaki (2015). 
the former approach. Concretely, I posit the following interrogative operator, which resides in the $\mathrm{C}$ position of interrogative CPs other than polar questions.

$$
?:=\lambda Q_{\langle s t, t\rangle} \cdot\left\{p \mid \exists p^{\prime} \in Q\left[p=\lambda w: \exists p \in Q\left[p=\operatorname{MAX}_{\text {inf }}(Q, w)\right] \cdot p^{\prime}\right]\right\}
$$

Given a Hamblin denotation, the operator returns a set of propositions, each of which carries the maximality presupposition that would amount to the uniqueness in case the set consists of logically independent atomic propositions.

Although I do not have a knockdown argument against the approach that localizes the presupposition to which, the approach based on an operator like (45) presents a viable alternative since it can be seen as a conservative extension of Dayal's (1996) analysis, preserving its various desirable consequences, such as the treatment of the exclusivity presupposition of alternative questions and the account of the negative island effect (Fox \& Hackl 2007, i.a.).

\section{Conclusions and an open issue}

In this paper, I pointed out that the projection patterns of the existential/uniqueness presupposition of $w h$-complements pose problems for the existing analyses by Dayal (1996) and Uegaki (2015), and argued that the patterns require the Partial Proposition Analysis, which treats the existential/uniqueness presupposition as triggered by the propositions corresponding to the answers of the question.

An important issue I left open is how the de relde dicto ambiguity of the whichphrase interacts with the uniqueness/existential presupposition. In the discussion above, my analysis of the UP assumed the de dicto construal (see, e.g., (30)). However, it is in principle possible in the Partial Proposition Analysis that the UP involves the de re construal, as exemplified below:

$$
\begin{aligned}
& \text { which student smokes } \rightsquigarrow \\
& \left\{\begin{array}{c}
\lambda w^{\prime}: \exists ! x\left[\text { student }_{w}(x) \wedge \text { smoke }_{w}(x)\right] . \text { smoke }_{w^{\prime}}(a), \\
\lambda w^{\prime}: \exists ! x\left[\text { student }_{w}(x) \wedge \mathbf{s m o k e}_{w}(x)\right] . \text { smoke }_{w^{\prime}}(b), \\
\lambda w^{\prime}: \exists ! x\left[\mathbf{s t u d e n t}_{w}(x) \wedge \mathbf{s m o k e}_{w}(x)\right] . \operatorname{smoke}_{w^{\prime}}(c)
\end{array}\right\} \quad \text { (de re UP) }
\end{aligned}
$$

What is important is that the UP construed de re is predicted to project to the matrix level even under the Partial Proposition Analysis. This has relevance to the problem concerning the prediction of the analysis in Section 3.2 that the UP is projected to the subject's beliefs with know. That is, the problem suggests that the full analysis has to include the predicates' preference/selection for de relde dicto. Specifically, the seeming incorrect prediction about the projection of UP to the subject's beliefs with know can be avoided if know prefers the de re construal of the UP while there is no similar preference in the case of be certain. This issue is left for future research. 
On the projection of the presupposition of embedded questions

\section{References}

Beaver, David. 2001. Presupposition and Assertion in Dynamic Semantics. Stanford, CA: CSLI Publications.

Champollion, Lucas, Ivano Ciardelli \& Floris Roelofsen. 2017. On questions and presuppositions in typed inquisitive semantics. Handout for the talk given at the 2nd workshop on Inquisitiveness Below and Beyond the Sentence Boundary (InqBnB).

Chemla, Emmanuel. 2009. Similarity: towards a unified account of scalar implicatures, free choice permission and presupposition projection. Ms., ENS Paris. http://www.emmanuel.chemla.free.fr/Material/Chemla-SIandPres.pdf.

Ciardelli, Ivano \& Floris Roelofsen. 2015. Inquisitive dynamic epistemic logic. Synthese 192(6). 1643-1687. doi:10.1007/s11229-014-0404-7.

Cremers, Alexandre. 2016. On the semantics of embedded questions. Paris: École Normale Supérieure $\mathrm{PhD}$ dissertation.

Dayal, Veneeta. 1996. Locality in Wh-quantification: Questions and Relative Clauses in Hindi. Dordrecht: Kluwer Academic Publishers. doi:10.1007/97894-011-4808-5.

Elliott, Patrick D., Andreea C. Nicolae \& Uli Sauerland. 2018. Who and what do who and what range over cross-linguistically? Ms., Leibniz-Zentrum Allgemeine Sprachwissenschaft. https://semanticsarchive.net/Archive/TM1Njk0M/ elliottNicolaeSauerland2018.pdf.

Fox, Danny. 2013. Mention-some readings of questions, class notes. MIT seminars.

Fox, Danny. 2018. Partition by exhaustification: Comments on Dayal 1996. In Uli Sauerland \& Stephanie Solt (eds.), Sinn und Bedeutung (SuB) 22, vol. 1 ZASPiL 60, 403-434. Berlin: Leibniz-Centre General Linguistics.

Fox, Danny \& Martin Hackl. 2007. The universal density of measurement. Linguistics and Philosophy 29(5). 537-586. doi:10.1007/s10988-006-9004-4.

Gallin, Daniel. 1975. Intensional and Higher-Order Modal Logic: With Applications to Montague Semantics. Amsterdam: North-Holland.

George, B.R. 2011. Question embedding and the semantics of answers. Los Angeles, CA: University of California, Los Angeles $\mathrm{PhD}$ dissertation.

Groenendijk, Jeroen \& Martin Stokhof. 1984. Studies on the semantics of questions and the pragmatics of answers. Amsterdam: University of Amsterdam $\mathrm{PhD}$ dissertation.

Hamblin, Charles L. 1973. Questions in Montague English. Foundations of Language 10(1). 41-53.

Heim, Irene. 1994. Interrogative semantics and Karttunen's semantics for know. In R. Buchalla \& A. Mittwoch (eds.), The Ninth Annual Conference and the Workshop on Discourse of the Israel Association for Theoretical Linguistics 
(IATL), Jerusalem: Academon.

Heim, Irene \& Angelika Kratzer. 1998. Semantics in Generative Grammar. Oxford: Blackwell Publishers.

Karttunen, Lauri. 1977. Syntax and semantics of questions. Linguistics and Philosophy 1. 3-44. doi:10.1007/bf00351935.

Katz, Jerrold J. \& Paul M. Postal. 1964. An Integrated Theory of Linguistic Descriptions. Cambridge, MA.: The MIT Press.

Lahiri, Utpal. 2002. Questions and Answers in Embedded Contexts. Oxford: Oxford University Press.

Nicolae, Andreea Cristina. 2013. Any questions? Polarity as a window into the structure of questions. Cambridge, MA: Harvard University PhD dissertation.

Rullmann, Hotze \& Sigrid Beck. 1998. Presupposition projection and the interpretation of which questions. In Devon Strolovitch \& Aaron Lawson (eds.), Semantics and Linguistic Theory (SALT) 8, 215-232.

Spector, Benjamin \& Paul Egré. 2015. A uniform semantics for embedded interrogatives: An answer, not necessarily the answer. Synthese 192(6). 1729-1784. doi:10.1007/s11229-015-0722-4.

Theiler, Nadine, Floris Roelofsen \& Maria Aloni. 2017. What's wrong with believing whether? In Semantics and Linguistic Theory (SALT) 27, 248-265. doi:10.3765/salt.v27i0.4125.

Theiler, Nadine, Floris Roelofsen \& Maria Aloni. 2018. A uniform semantics for declarative and interrogative complements. Journal of Semantics 35(3). 409466. doi:10.1093/jos/ffy003.

Uegaki, Wataru. 2015. Interpreting questions under attitudes. Cambridge, MA: Massachusetts Institute of Technology PhD dissertation.

Uegaki, Wataru. to appear. The semantics of question-embedding predicates. Language and Linguistics Compass .

Xiang, Yimei. 2016. Interpreting questions with non-exhaustive answers. Cambridge, MA: Harvard University PhD dissertation.

Wataru Uegaki

Leiden University Centre for Linguistics

Van Wijkplaats 4, 2311 BX Leiden

The Netherlands

w.uegaki@hum.leidenuniv.nl 